\title{
Physical limits of semiconductor laser operation: A time-resolved analysis of catastrophic optical damage
}

Ziegler, Mathias; Hempel, Martin; Larsen, Henning Engelbrecht; Tomm, Jens W.; Andersen, Peter E.; Clausen, Sønnik; Elliott, Stella N.; Elsaesser, Thomas

Published in:

Applied Physics Letters

Link to article, DOI:

10.1063/1.3463039

Publication date:

2010

Document Version

Publisher's PDF, also known as Version of record

Link back to DTU Orbit

Citation (APA):

Ziegler, M., Hempel, M., Larsen, H. E., Tomm, J. W., Andersen, P. E., Clausen, S., Elliott, S. N., \& Elsaesser, T. (2010). Physical limits of semiconductor laser operation: A time-resolved analysis of catastrophic optical damage. Applied Physics Letters, 97(2), 021110. https://doi.org/10.1063/1.3463039

\section{General rights}

Copyright and moral rights for the publications made accessible in the public portal are retained by the authors and/or other copyright owners and it is a condition of accessing publications that users recognise and abide by the legal requirements associated with these rights.

- Users may download and print one copy of any publication from the public portal for the purpose of private study or research.

- You may not further distribute the material or use it for any profit-making activity or commercial gain

- You may freely distribute the URL identifying the publication in the public portal 


\title{
Physical limits of semiconductor laser operation: A time-resolved analysis of catastrophic optical damage
}

\author{
Mathias Ziegler, ${ }^{1, a)}$ Martin Hempel, ${ }^{1}$ Henning E. Larsen, ${ }^{2}$ Jens W. Tomm, ${ }^{1, b)}$ \\ Peter E. Andersen, ${ }^{2}$ Sønnik Clausen, ${ }^{3}$ Stella N. Elliott, ${ }^{4}$ and Thomas Elsaesser ${ }^{1}$ \\ ${ }_{1}^{1}$ Max-Born-Institut für Nichtlineare Optik und Kurzzeitspektroskopie, Max-Born-Str. 2 A, \\ 12489 Berlin, Germany \\ ${ }^{2}$ Department of Photonics Engineering, Technical University of Denmark, DK4000 Roskilde, Denmark \\ ${ }^{3}$ Ris $\phi$ DTU, Technical University of Denmark, DK4000 Roskilde, Denmark \\ ${ }^{4}$ Cardiff School of Physics and Astronomy, Cardiff University, The Parade, Cardiff CF24 3AA, \\ United Kingdom
}

(Received 17 May 2010; accepted 20 June 2010; published online 15 July 2010)

\begin{abstract}
The early stages of catastrophic optical damage (COD) in $808 \mathrm{~nm}$ emitting diode lasers are mapped by simultaneously monitoring the optical emission with a $1 \mathrm{~ns}$ time resolution and deriving the device temperature from thermal images. COD occurs in highly localized damage regions on a 30 to $400 \mathrm{~ns}$ time scale which is determined by the accumulation of excess energy absorbed from the optical output. We identify regimes in which COD is avoided by the proper choice of operation parameters. (C) 2010 American Institute of Physics. [doi:10.1063/1.3463039]
\end{abstract}

Early work on diode laser degradation has attributed catastrophic optical damage (COD) to a thermal runaway due to a positive feedback between elevated temperatures at the laser facet and the increase in the temperature-dependent absorption of laser light. ${ }^{1,2}$ The resulting temperature increase eventually leads to a local melting of the semiconductor lasers surface and a subsequent propagation of the damage front into the device. In Ref. 2, the intrinsic interband absorption at the fundamental band gap has been considered the starting point of COD. Later, it was shown that extrinsic effects such as surface recombination and the creation of structural defects are involved in the absorption and temperature rise as well and eventually dominate. ${ }^{3-5}$ Specific spatially and temporally resolved experiments ${ }^{6-8}$ on the build-up of COD have remained scarce and, as a result, theoretical attempts to describe COD kinetics, for instance as a microexplosion, ${ }^{1,3,9,10}$ have not been experimentally confirmed.

In this letter, we report an analysis of early stages of $C O D$ during single-pulse excitation. We monitor the time evolution of the emitted power with a temporal resolution of 1 ns. Simultaneously, thermal images of the devices are recorded with a spatial resolution of $\sim 5 \mu \mathrm{m}$. While temporally integrating over the entire pulse, thermography gives evidence of the presence of a local thermal runaway manifested in a thermal flash of Planck's radiation. Structural information on local damage is gained by microscopy and incorporated into a model describing the energy balance of the COD process.

A batch consisting of 14 commercial devices of gainguided $808 \mathrm{~nm}$ emitting AlGaAs-based broad area quantum well high-power diode lasers is investigated. Emitter stripe width and cavity length are 50 and $1400 \mu \mathrm{m}$, respectively. Facets are coated with standard antireflective and highreflective dielectric mirrors. The devices are specified for $\mathrm{cw}$

\footnotetext{
${ }^{\text {a) }}$ Present address: BAM Federal Institute for Materials Research and Testing, 12200 Berlin, Germany.

b) Author to whom correspondence should be addressed. Electronic mail: tomm@mbi-berlin.de.
}

operation with an output power of $0.5 \mathrm{~W}$ at $0.6 \mathrm{~A}$ and a threshold current of $0.2 \mathrm{~A}$. The lasers were driven with single current pulses (Picolas LDP-V50-100V3 current driver) between 2 and $50 \mathrm{~A}$ with pulse lengths of $50 \mathrm{~ns}$ to $100 \mu \mathrm{s}$ (rise times $\sim 13 \mathrm{~ns}$ ). The temperature of the heat sink is $(23.0 \pm 0.1){ }^{\circ} \mathrm{C}$.

A dichroic mirror tilted by $40^{\circ}$ relative to the front facet of the investigated laser transmits the thermal radiation and reflects the fundamental laser emission. The thermal radiation is detected with a CEDIP Titanium $560 \mathrm{M}$ thermocamera equipped with an optical bandpass filter for the 4.3-6.0 $\mu \mathrm{m}$ spectral range and a microscope lens (spatial resolution $4.7 \mu \mathrm{m} / \mathrm{pixel}$ ). The time evolution of laser emission is recorded with a reverse-biased $\mathrm{Si}$ pin photodiode (Thorlabs DET10A/M, rise time $\sim 1 \mathrm{~ns}$ ) attached to a $2 \mathrm{GHz}$ oscilloscope (Agilent Infiniium). Data acquisition works as follows: a delay generator triggers the acquisition of a thermal image with an integration time of $7 \mu \mathrm{s}$. The thermocamera generates a signal which is used to trigger the single current pulse starting $3 \mu \mathrm{s}$ after the thermocamera resulting in an effective integration time of $4 \mu \mathrm{s}$. The background is subtracted from all camera images. Temperature calibration was performed between 23 and $200{ }^{\circ} \mathrm{C}$. Temperatures above this range are estimated according to Planck's law, taking into account the camera's spectral responsivity and transmittances of the optical elements. L-I-V characteristics were measured for all devices and their linear range was used to calibrate the photodiode signal. On a second channel the current is monitored.

COD becomes effective at high currents. Figure 1 documents the resulting structural alterations for two individual devices, each of them having been driven by a single current pulse. In Figs. 1(a), 1(e), 1(f), and 1(j) the lateral profiles of the emission nearfields are shown. Device damage is manifested in decent changes in the intensity distributions [Figs. $1(\mathrm{~b}), 1(\mathrm{e}), 1(\mathrm{~g})$, and $1(\mathrm{j})]$. There is a clear spatial correlation between the drop of output power in the nearfield [Figs. 1(b) and $1(\mathrm{~g})]$ and the facet damage patterns detected by optical microscopy [Figs. 1(c) and 1(h)]. The latter act as local 


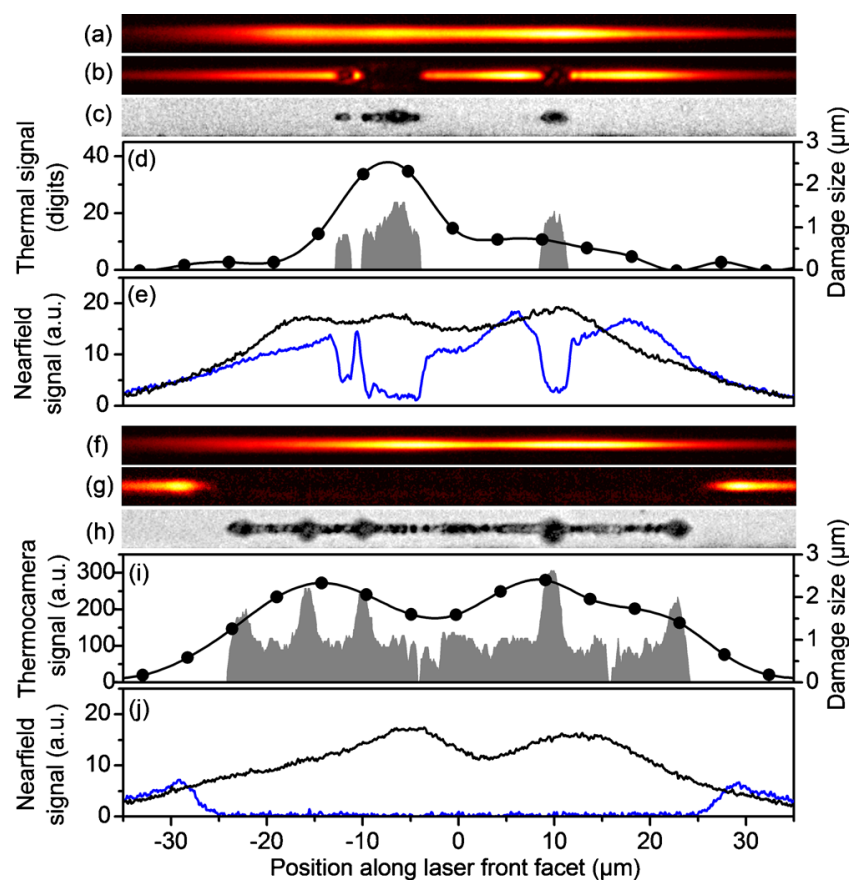

FIG. 1. (Color online) Data sets for two representative devices that underwent COD at low current [upper panels (a)-(e)] and at elevated current [panels (f)-(j)]. [(a), (b), (f), and (g)] Spatial distribution of laser emission at the front facet (optical nearfield) along the lateral facet dimension measured with a spatial resolution of $\sim 1 \mu \mathrm{m}$ for injection currents of [(a), (b), (f), and $(\mathrm{g})] 0.5 \mathrm{~A}$. The panels (b) and (c) and (g) and (h) were recorded after COD. [(c) and (h)] Microscope images of the front facets acquired after COD showing the dark damaged regions. [(d) and (i)] Spatial profiles of the emitted thermal power (solid lines and symbols) derived from Fig. 2 (right panel) and damage size as derived from Figs. 1(c) and 1(h). [(e) and (j)] Spatial profiles of emitted laser power before (black lines) and after COD (blue lines).

sources of thermal radiation [Figs. 1(d) and 1(i)] resulting in the observed thermal flashes. ${ }^{8}$

Figure 2 shows current and emission power transients recorded for four devices. Driving currents between 4 and $25 \mathrm{~A}$ are chosen. While no degradation is observed for $4 \mathrm{~A}$, COD occurs at higher currents and leads to a collapse of power on a nanosecond time scale. With increasing current, the decrease in power sets in at earlier times and the power decay times (1/e) are reduced from $\sim 400$ to $30 \mathrm{~ns}$. The occurrence of COD is evident from the observation of a thermal flash recorded with the calibrated thermocamera [right panels in Figs. 2(b)-2(d)]. The microscope images in Figs. 1(c), 1(d), 1(h), and 1(i) give quantitative information on the damaged facet area, which allows us to estimate the local temperatures given in Figs. 2(b)-2(d). Such temperatures are on the order of the melting temperature of GaAs of $1240{ }^{\circ} \mathrm{C}$.

We varied both the amplitude and the duration of the individual current pulses. The data is summarized in a twodimensional plot [Fig. 3(a)]. The solid symbols represent individual measurements in which COD and a thermal flash is observed. The events are placed at the average optical output power $P_{\text {out }}$ before COD (bottom abscissa scale) which corresponds to the current amplitude given on top, and at the onset time of COD $\left(t_{\mathrm{COD}}\right)$ on the right ordinate scale. $t_{\mathrm{COD}}$ is defined as the time at which the output power starts to decrease; see arrows in Fig. 2. There is a clear correlation in the data set, showing shorter $t_{\mathrm{COD}}$ times for higher output powers and current amplitudes. The open symbols in Fig. 3(a) represent devices in which COD was absent, as is evident from

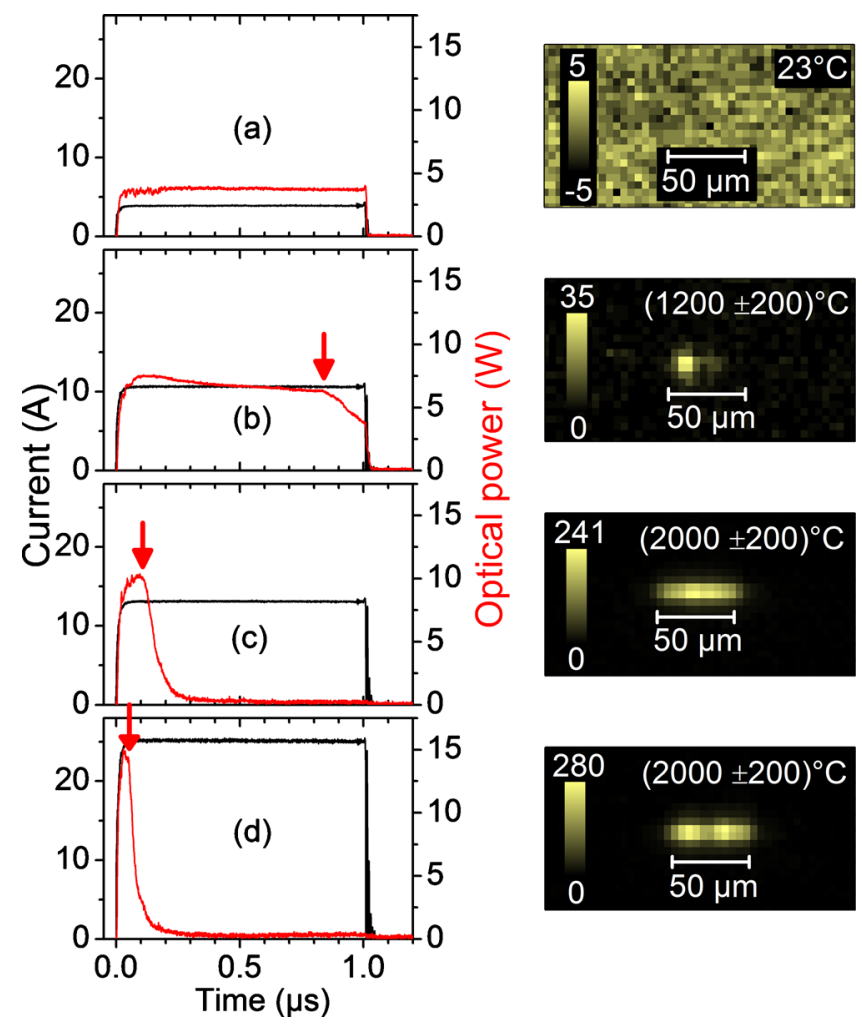

FIG. 2. (Color online) [(a)-(d)] Current and emission power transients from four devices: diode laser current (black lines) and optical output power (red lines) as a function of time. The onset of COD (at time $t_{\mathrm{COD}}$ ) is marked by arrows. The right panel shows the corresponding thermal images recorded during the current pulse. Estimated maximal temperatures of the thermal flashes are included.

their essentially homogeneous thermal images with temperatures close to the ambient temperature [right panel in Fig. $2(a)]$ and the absence of any degradation effects. Such measurements are placed at the respective output power and current amplitude and duration of the current injection pulse (left ordinate scale). The overall set of results defines a borderline between a parameter range in which COD occurs [orange area in Fig. 3(a)] and a region without COD (blue area). The blurred region in-between reflects the scattering of individual device properties within our sample set. The dashed line shows the "square-root law" that has been originally discovered for devices with lower emission powers. 1,11 Our data confirm this finding as long as heat dissipation is negligible; i.e., for short $t_{\mathrm{COD}}$ or pulse durations.

The data in Fig. 3(a) show the time from the start of the current pulse to $t_{\mathrm{COD}}$ to vary over two orders of magnitude depending on operation current. We now discuss the physical mechanisms that cause the first phase of COD to take place on the nanosecond time scale. COD is characterized by an initial local melting at the front facet induced by thermal excess energy that is provided by a local absorption of optical power in the device. On the short timescale of our experiments of $1 \mu \mathrm{s}$, the loss of excess energy due to heat conduction into other parts of the device structure is negligible and, thus, the time-integral of absorbed optical power can directly be correlated with the energy required for melting. It should be noted that the well-known propagation of damage into the device, the second phase of COD, occurs on a slower microsecond to millisecond time scale not considered here. ${ }^{6,12-14}$ 


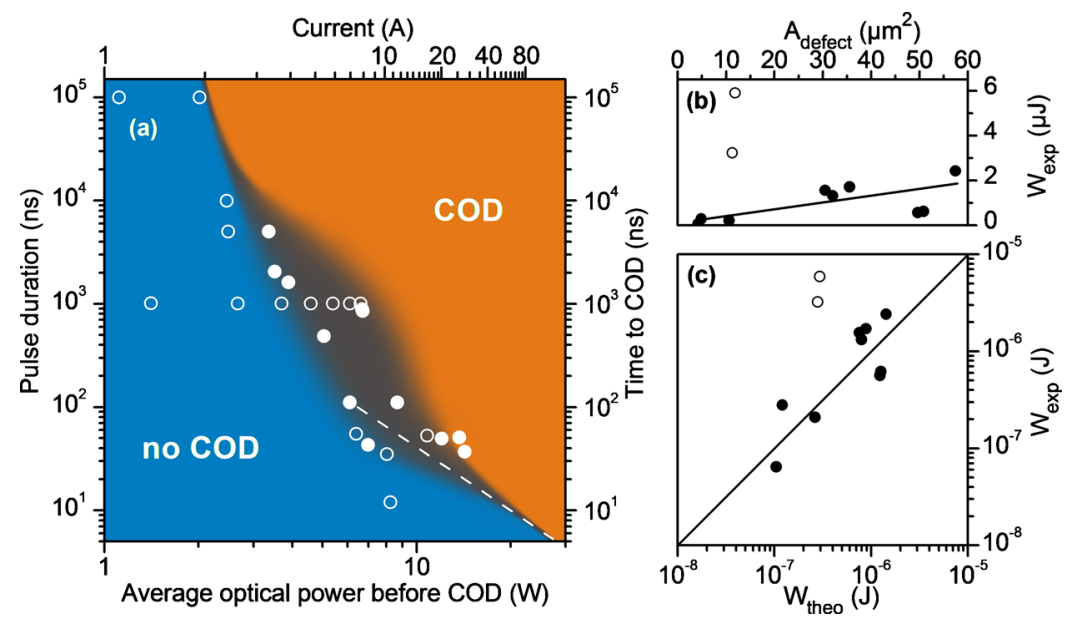

FIG. 3. (Color online) (a) COD diagram showing the regions of COD (filled circles) and no COD (open circles) occurrence. The occurrence of COD was derived from an analysis of thermal images such as displayed in Fig. 2 (right panel). The borderline (colored gray) is blurred because of the randomness in filamentation and scatter in device properties. The dashed line represents the "square-root law" that has been originally discovered for devices with smaller emission powers (Refs. 1 and 11). (b) Absorbed output energy $W_{\text {exp }}$ calculated from the experimental transients vs damaged area on the front facet (symbols). The solid line is to guide the eye. (c) Absorbed output energy $W_{\text {exp }}$ as a function of the calculated thermal energy $W_{\text {theo }}$ required to heat the damaged device volume to the melting point (symbols)
To validate this picture, we calculate the energy $W_{\text {theo }}$ required to heat the damaged device volume to the melting point and correlate it with the absorbed optical energy $W_{\exp }$ that is derived from our time-resolved data such as shown in Fig. 2. The required excess energy is given by $W_{\text {theo }}$ $=V_{\text {defect }} \rho c\left(T_{\text {melt }}-T_{0}\right)$, where $V_{\text {defect }}=A_{\text {defect }} z_{0}$ is the volume of the damaged part of the device with the defect area $A_{\text {defect }}$ taken from the microscope images in Fig. 1. A depth $z_{0}$ $=15 \mu \mathrm{m}$ eventually provided the best fit to the data and corresponds to defect propagation velocities as determined in Ref. 6 The other parameter values for GaAs are the specific heat $c=0.33 \mathrm{~J} /(\mathrm{gK})$, the mass density $\rho=5.33 \mathrm{~g} / \mathrm{cm}^{3}$, and melting temperature $T_{\text {melt }}=1240{ }^{\circ} \mathrm{C}\left(T_{0}=23{ }^{\circ} \mathrm{C}\right)$. The absorbed optical energy $W_{\text {exp }}$ which is accumulated in $V_{\text {defect }}$ is calculated from the measured power transients $P(t)$ according to

$$
W_{\exp }=\int_{t_{\mathrm{COD}}}^{t_{2}}\left[P\left(t_{\mathrm{COD}}\right)-P(t)\right] d t .
$$

$P\left(t_{\mathrm{COD}}\right)$ is the output power of the laser reached at the onset time of COD, $t_{\mathrm{COD}}$, and $P(t)$ is the time dependent output power at times $t>t_{\mathrm{COD}}$. The upper integration limit $t_{2}$ represents the time at which the output power has dropped to $P\left(t_{\mathrm{COD}}\right) / \mathrm{e}^{2}=0.135 P\left(t_{\mathrm{COD}}\right)$ or the pulse has ended. In principle, the absorbed energy should be calculated from the internal optical power rather than from the output power $P$. For the low reflectivity of $R=0.1$ of the outcoupling facet, however, both have very similar values. Our approach also neglects the small amount of energy required to reach the onset of COD.

Results of this analysis are presented in Figs. 3(b) and 3(c) where each symbol represents an individual device. Figure 3(b) shows an essentially linear correlation between $W_{\text {exp }}$ and $A_{\text {defect }}$, in line with the anticipated damage mechanism driven by $W_{\text {exp }}$. Figure 3(c) gives the reabsorbed energy $W_{\text {exp }}$ and the melting energy $W_{\text {theo }}$ for each device in a doublelogarithmic plot. We find a linear correlation with $W_{\text {exp }}$ $\approx W_{\text {theo }}$ for the majority of the devices [(solid symbols in Fig. 3(b)], covering nearly two orders of magnitude on the respective energy scale. This strongly supports the COD scenario outlined above and characterizes COD as a mainly thermal effect. The two open symbols in Figs. 3(b) and 3(c) which lie substantially above the correlation represent devices which were driven with longer current pulses of 5 and $100 \mu \mathrm{s}$ and displayed COD in the microsecond time domain. On this longer time scale, heat transport from the damaged volume into other parts of the device removes part of the deposited excess energy $W_{\text {exp }}$, i.e., COD is induced with a fraction of $W_{\text {exp }}$ only.

In conclusion, the time resolved experiments presented here demonstrate nanosecond time intervals to COD. This time scale is determined by the accumulation time of excess energy which is absorbed from the optical output and results eventually in a local melting of the device. Our work identifies a regime in which COD of high-power semiconductor lasers is avoided by the proper choice of operation parameters, allowing the ultimate limit of semiconductor laser operation to be reached. Our results also serve as a benchmark for future modeling of early COD dynamics and the transition between the two time regimes identified.

Funding by the European Commission within the project WWW.BRIGHTER.EU under Contract No. IST2005-035266 is acknowledged.

${ }^{1}$ P. G. Eliseev, J. Lumin. 7, 338 (1973).

${ }^{2}$ C. H. Henry, P. M. Petroff, R. A. Logan, and F. R. Merritt, J. Appl. Phys. 50, 3721 (1979).

${ }^{3}$ W. Nakwaski, J. Appl. Phys. 57, 2424 (1985).

${ }^{4}$ G. Chen and C. L. Tien, J. Appl. Phys. 74, 2167 (1993).

${ }^{5}$ R. Schatz and C. G. Bethea, J. Appl. Phys. 76, 2509 (1994).

${ }^{6}$ J. H. Jacob, R. Petr, M. A. Jaspan, S. D. Swartz, M. T. Knapczyk, and A. M. Flusberg, Proc. SPIE 7198, 71981A1 (2009).

${ }^{7}$ M. Ziegler, J. W. Tomm, T. Elsaesser, C. Matthiesen M. BouSanayeh, and P. Brick, Appl. Phys. Lett. 92, 103514 (2008).

${ }^{8}$ M. Ziegler, J. W. Tomm, D. Reeber, T. Elsaesser, U. Zeimer, H. E. Larsen, P. M. Petersen, and P. E. Andersen, Appl. Phys. Lett. 94, 191101 (2009).

${ }^{9}$ P. G. Eliseev, Prog. Quantum Electron. 20, 1 (1996).

${ }^{10}$ W. R. Smith, J. Appl. Phys. 87, 8276 (2000).

${ }^{11}$ F. Kappeler, K. Mettler, and K. H. Zschauer, Pulsed-power performance and stability of $880 \mathrm{~nm}$ GaAlAs/GaAs oxide-stripe lasers (IEEE, New York, 1982), Vol. 129(6), p. 256.

${ }^{12}$ B. W. Hakki and F. R. Nash, J. Appl. Phys. 45, 3907 (1974).

${ }^{13}$ O. Ueda, K. Wakao, S. Komiya, A. Yamaguchi, S. Isozumi, and I. Umebu, J. Appl. Phys. 58, 3996 (1985)

${ }^{14}$ K. H. Park, J. K. Lee, D. H. Jang, H. S. Cho, C. S. Park, K. E. Pyun, J. Y. Jeong, S. Nahm, and J. Jeong, Appl. Phys. Lett. 73, 2567 (1998). 